\title{
La preposición àđó en el griego del Nuevo Testamento. Algunos casos de controversia
}

\author{
Marta Merino Hernández ${ }^{1}$
}

Recibido: 18 de octubre de 2016 / Aceptado: 9 de enero de 2017

Resumen. Una adecuada comprensión del significado de las preposiciones en contexto es vital para la exégesis. En efecto, el debate exegético ha girado, en ocasiones, en torno al valor de una preposición en concreto. Sin embargo, siguen existiendo casos de desacuerdo en torno al sentido del morfolexema ả en diferentes pasajes del NT, tanto en las traducciones y los principales diccionarios de griego clásico y neotestamentario como en algunas gramáticas y estudios. Este trabajo examinará pasajes del NT en los que el valor agentivo (de predicación pasiva) y el causal de ả $\pi_{\text {ó }}$ son erróneamente identificados entre sí o con otras funciones como las de separación, procedencia o intermediación. Tratará de dilucidar, por tanto, cuál es el ámbito significativo de la preposición en cada caso siguiendo la metodología del Diccionario Griego-Español del Nuevo Testamento (DGENT), (Córdoba 2000-) de J. Mateos (†) - J. Peláez J. \& GASCO, cuyo marco teórico seguiremos, así como dentro del eje o continuum conceptual que abarca las nociones de Causa y Agente e Intermediario. En definitiva, este artículo muestra cómo la aplicación del método de análisis semántico del DGENT, que supone nueva forma de tratar los lexemas, constituye un avance en el examen y la sistematización de los valores semánticos de la preposición àmó en el griego del corpus del NT, ya que permite analizar con precisión la preposición en contexto, arrojando luz sobre algunos pasajes neotestamentarios en los que su sentido ha sido largamente discutido por los investigadores.

Palabras clave: preposición $\alpha$ đó; valor agentivo, causal y de intermediación; semántica; sintaxis; Nuevo Testamento.

\section{[en] The ámó preposition in the Greek New Testament. Somes cases of controversy}

\begin{abstract}
A proper understanding of the meaning of prepositions in context is vital for exegesis. Indeed, the exegetical debate has been sometimes about the value of a particular preposition. However, there are still cases of disagreement about the meaning of à $\pi$ ó morpholexeme in different passages of the New Testament in translations, major classical and New Testament Greek dictionaries as well as in some grammars and studies. This paper will analyze passages from the New Testament where the agentive value (passive predication) and the causal use of $\dot{\alpha} \pi$ are misidentified with each other or with other functions such as separation, origin or intermediation. We will try to figure out, therefore, what is the significant scope of the preposition in each case, following the methodology of the Greek-Spanish Dictionary of the New Testament (DGENT), (Córdoba 2000-) of J. Mateos (†) - J. Pelaez J. \& GASCO, whose theoretical framework we will follow, as well as within the axis or conceptual continuum that encompasses the notions of cause, agent and intermediary. In short, this article shows how the application of the DGENT method of semantic analysis, representing new way of treating the lexemes, is a progress in the analysis and systematization of the semantic values of the $\dot{\alpha} \pi$ ó preposition in the corpus of the Greek of the NT because it allows to accurately analyze the preposition in context, shedding light on some New Testament passages in which the meaning has long been discussed by researchers.
\end{abstract}

$1 \quad$ Universidad de Córdoba. Dpto. de Ciencias de la Antigüedad

E-mail: martmerino1@hotmail.com 
Keywords: ảjó preposition; agentive, causal and intermediation values; semantics; syntax; New Testament.

Sumario. I. Introducción. II. La preposición ảđó. Algunas observaciones preliminares. II.1. La

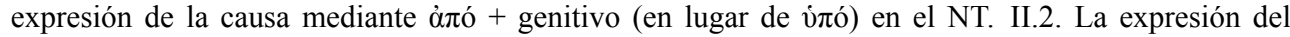
agente mediante $\dot{\alpha} \hat{o}^{+}+$genitivo (en lugar de $\dot{\pi}$ ó) en el NT. III. El continuum conceptual agente-causaintermediario. IV. Algunos casos de controversia respecto al valor de la preposición ảंó en el NT

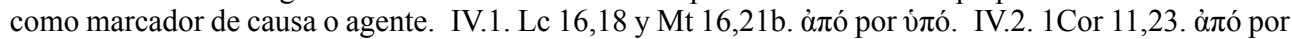
$\pi \alpha \rho \alpha ́$. IV.3. Lc 12,57. V. Conclusiones.

Cómo citar: Merino Hernández, M. (2017) La preposición ả Algunos casos de controversia, en Cuadernos de Filología Clásica. Estudios griegos e indoeuropeos $27,31-47$

\section{Introducción}

Una adecuada comprensión del significado de las preposiciones en contexto es vital para la exégesis. En efecto, el debate exegético ha girado, en ocasiones, en torno al valor de una preposición en concreto. Sin embargo, como veremos, siguen existiendo casos de desacuerdo en torno al sentido del morfolexema ỏ ó en diferentes pasajes del NT, tanto en las traducciones y los principales diccionarios de griego clásico y neotestamentario como en algunas gramáticas y estudios.

En este contexto, el presente trabajo examinará pasajes neotestamentarios en los que el valor agentivo (de predicación pasiva) y el causal del morfolexema $\alpha$ ó son erróneamente identificados entre sí o con otras funciones como las de separación, procedencia o intermediación. Tratará de dilucidar, por tanto, cuál es el ámbito significativo del morfolexema en cada caso siguiendo la metodología del $D G E N T^{2}$, cuyo marco teórico seguiremos en estas páginas ${ }^{3}$.

Con frecuencia, las preposiciones son examinadas de manera simplista e incluso apriorística, es decir, tratándose de forma aislada, sin profundizar en su vinculación con el contexto en el que se encuentran (Wallace 1996: 359). Este último, como veremos, es un elemento imprescindible para dilucidar el sentido de esta clase de morfolexemas, cuya dificultad de estudio, tanto sintáctico como semántico, se debe, por una parte, a que se trata de una categoría gramatical muy compleja, ambigua y polisémica, expuesta, a menudo, a sutiles matices semánticos; por otra, a que

\footnotetext{
Mateos (†), Peláez \& GASCO (2000-), DGENT. Fasc. 1-5, Córdoba, El Almendro.
}

El análisis de la preposición ỏ ó en contexto que centra este trabajo se basa en el empleo sistemático de la metodología de análisis utilizada por el DGENT (2007 s.v. àjó: 789-829) para la redacción de sus lemas; esta se fundamenta en cuatro principios básicos que dan lugar a los principales avances de este léxico:

a) Diferenciación sistemática entre significado y traducción en la redacción de los lemas;

b) elaboración de la definición del significado lexical de los lexemas, es decir, de una descripción del mismo a la que acompañará también la traducción correspondiente. Por lo demás, no solo se da una definición y traducción del significado base, sino también de los diferentes sememas o acepciones del lexema estudiado;

c) mención del factor o factores contextuales como elemento determinante del cambio semántico de los lexemas de manera individualizada y

d) cotejo de todos los contextos en que se encuentra el lexema dentro del corpus neotestamentario.

De este modo, en el caso de àđó, el DGENT (2007 s.v. ஷ̇ंó: 789-829) ha verificado que existen once acepciones diferentes en el corpus neotestamentario y que es posible explicar por qué el morfolexema cambia de un significado a otro atendiendo a los diferentes usos de este en contexto. 
su sistema en griego es complementario del de los casos, por lo que representa un terreno de estudio a medio camino entre lo gramatical y lo lexical. Sin embargo, cada preposición griega posee un significado lexical ${ }^{4}$ que es precisado y desarrollado de manera más amplia por el contexto en que aparece (Harris 2012: 27).

Así, por una parte, las preposiciones determinan la relación entre los términos involucrados y, por otra, estos suscitan, en cierta medida, la elección y el valor de la adposición que los une. De este modo, en la delimitación de la noción que expresa una preposición confluyen tres elementos: raíz preposicional, caso del sustantivo con el que compone el sintagma preposicional y contexto. Se debe comenzar, pues, con la idea expresada por la raíz — aunque esta debe matizarse con la función básica del caso nominal en articulación con el contexto inmediato (Alexandre 2016: 196) ${ }^{5}$. El significado lexical de un morfolexema preposicional servirá, además, para entender su extensa gama de sentidos derivados tanto abstractos como figurados (Alexandre 2016: 196), la cual, según P. Bortone (2010: 85), consiste en una red de extensiones metafóricas surgidas a partir de este ${ }^{6}$. Por consiguiente, para emprender el análisis de los valores de esta clase de morfolexemas es preciso, desde nuestra perspectiva, partir de un enfoque metodológico principalmente semántico (sin prescindir del aspecto morfosintáctico), así como estructural ${ }^{7}$.

En este sentido, es necesario señalar que el enfoque que presentan algunos de los principales diccionarios de griego clásico y neotestamentario en lo que respecta al análisis de las preposiciones, a pesar de los progresos realizados en materia lingüística, resulta aún limitado desde el punto de vista semántico, como veremos más adelante en el caso de $\alpha \pi^{\prime}{ }^{8}$.

$4 \quad$ Es decir, aquel del que podemos deducir el resto de acepciones surgidas por influjo contextual. Por nuestra parte, adoptamos la definición de significado lexical que dan Louw \& Nida: «Conjunto de rasgos semánticos (componentes semánticos o semas) de un término» (Louw \& Nida 1988: Introducción. XVI). El significado contextual es «aquel que resulta de adiciones efectuadas al núcleo sémico y / o de alteraciones producidas en el núcleo sémico mismo o en la figura nuclear de un lexema por los clasemas y semas ocasionales, y que exige una nueva definición. Equivale a “acepción”» (J. Peláez 1996: 163).

5 Así, el significado lexical de la mayor parte este tipo de morfolexemas está relacionado con la posición de movimiento y pausa. Las preposiciones que se articulan con acusativo representan el movimiento o dirección hacia; las preposiciones con genitivo, como ảió, indican el movimiento o dirección a partir de. Las preposiciones que se construyen con dativo denotan reposo o ausencia de movimiento (Alexandre 2016: 196).

6 De este modo, según el modelo de análisis formulado por la semántica cognitiva, es posible explicar el vínculo entre los distintos significados de una preposición, ya que entiende la polisemia preposicional como una cadena de extensiones semánticas de cuyo núcleo, formado por su noción espacial, se originarían los demás sentidos, lo que elucida el hecho de que una adposición pueda, según el contexto, adquirir significados no solo heterogéneos sino, a veces, incompatibles sincrónicamente, así como desarrollarlos diacrónicamente. $\mathrm{O}$ el de que la misma noción abstracta puede ser expresada por varias preposiciones de diferente sentido espacial (Galichet 1947: 46-47 y Bortone 2010: 40-41). Las mismas conclusiones pueden extraerse del modelo teórico de Mateos (1989: 8-9) que seguiremos en estas páginas, y para quien las diversas acepciones de un término (o sememas) son consecuencia de adiciones efectuadas al núcleo sémico y/o de alteraciones que se producen en el núcleo mismo de un lexema por semas de distinta índole. Por consiguiente, tanto la hipotética falta de sentido de las preposiciones como la presumible falta de relación entre su amplia gama de significados resultan del todo inadmisibles.

7 Este artículo forma parte de los trabajos preparatorios para la redacción del diccionario que el grupo GASCO (Grupo de Análisis Semántico del Área de Filología Griega de la Universidad de Córdoba) lleva a cabo, dentro del marco del Proyecto de Investigación «Diccionario Griego-Español del Nuevo Testamento», Modalidad 4.1. 2016. Universidad de Córdoba.

8 Siguiendo la metodología del DGENT(2000-2012), el primer paso para el estudio del valor de la preposición ả los pasajes que centrarán nuestra atención en este artículo es el examen de la misma en los principales diccionarios de griego clásico y neotestamentario. Nos referimos a los de H.G. Liddell \& R. Scott \& al. (LSJ), (1996 ${ }^{15}$, s.v. ḋó),

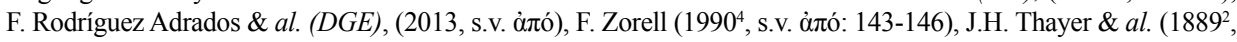

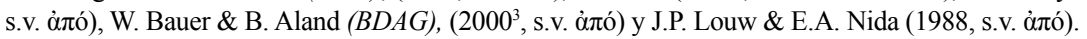


La primera parte de este artículo examinará las características propias de esta adposición, centrándose en su codificación de la causa y de la agentividad (con predicación pasiva) en sustitución de viđó en el NT; la segunda, examinará los rasgos y factores que, según la semántica funcional y cognitiva, permiten diferenciar ambas nociones no solo entre sí, sino de otros valores como la intermediación dentro del continuum conceptual agente-causa-intermediario; la tercera supondrá el núcleo de este estudio: el examen de la adposición que nos ocupa y la elucidación de su significado en diversos versículos del NT en los que este es motivo de diferentes interpretaciones por parte de traducciones, diccionarios, gramáticas y estudios, mediante el estudio del factor contextual y los rasgos semánticos propios de cada valor; y por último, se presentarán las conclusiones de esta investigación, examinándose las perspectivas que se abren para el estudio de la sintaxis y la semántica preposicional.

\section{La preposición ò $\pi$ ó. Algunas observaciones preliminares}

El morfolexema preposicional àđó ocupa el séptimo lugar en cuanto a su frecuencia (646) respecto al resto de las preposiciones denominadas propias ${ }^{9}$ en el NT (Harris 2012: 57) y tuvo un amplio uso en todos los períodos (Adrados 2005: 242). Rige sólo genitivo y denota la separación o el punto de partida en el espacio y en el tiempo ${ }^{10}$,

El examen de àjó por parte de estos seis diccionarios revela un claro desacuerdo entre los diferentes léxicos acerca de los sentidos de la preposición. En suma, constata que la falta de distinción entre significado base (o lexical) y contextual es un defecto básico tanto de la lexicografía tradicional como de la actual, si exceptuamos el léxico de Louw \& Nida. No obstante, es indispensable recordar que, aunque fueron escritos en un tiempo en el que la morfología y la sintaxis, más que la semántica, centraba el interés de los lexicográfos, estos diccionarios continúan siendo herramientas muy valiosas como léxicos de uso que ofrecen equivalentes de traducción en una lengua moderna o latina acompañados de información gramatical (Peláez

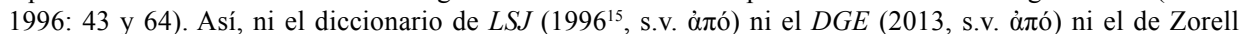

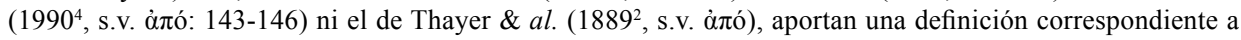
cada acepción, pues carecen de una metodología de análisis semántico que permita establecer y describir tanto el sentido de los lexemas como el de sus diversas acepciones. Ello da lugar a frecuentes inexactitudes en su análisis de los contextos, así como a incoherencias. También se detecta poca sistematicidad en el análisis de los vocablos, haciendo que los diferentes apartados no correspondan, por lo general, a una acepción diferente, sino solo a un uso sintáctico distinto. En general, la organización del lema en estos diccionarios parece estar determinada, con frecuencia, por principios de clasificación gramatical o sintáctica, aunque los autores no justifican el enfoque seguido para fijar las diferentes acepciones; tampoco explican cuál es el factor contextual que provoca el cambio de sentido del morfolexema.

El mérito del diccionario de Louw \& Nida (1988, s.v. à de la semántica a la lexicografía, abriendo una nueva vía en esta materia. Sin embargo, su taxonomía para el análisis de los lexemas por campos semánticos no logra organizar el lema de manera uniforme, ya que los criterios expuestos en su introducción no se aplican de manera sistemática. Por tanto, su análisis de ỏ $\pi$ ć carece a menudo de precisión, ya que los autores no explican cuál es el factor contextual que incide en el cambio de significado del morfolexema en contexto. Por lo demás, aunque este léxico proporciona una definición de cada una de las acepciones, peculiaridad poco frecuente en los diccionarios bilingües, estas suelen ser imprecisas o tautológicas. A esto hay que añadir que este diccionario no diferencia entre significado lexical y contextual, pues carece de un método uniforme para explicitar el significado de los lexemas (Peláez 1996: 63).

9 Se trata de preposiciones propiamente dichas, las cuales fueron en su origen adverbios independientes que posteriormente adquirieron uso preverbial y adpositional a través de un proceso de gramaticalización, aunque todavía evidencian vestigios de su función adverbial original. Estas suelen aparecer como elemento inicial o prefijo en algunos sustantivos, adjetivos y adverbios, así como en verbos, funcionando en este caso como preverbios; se caracterizan por ser bisilábicas o monosilábicas.

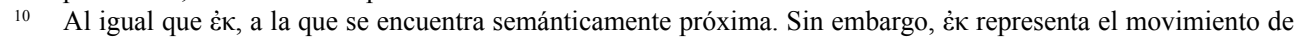
dentro hacia afuera de una superficie u objeto. 
expresando el movimiento desde el borde o superficie de un objeto. De este significado básico se derivan, metafóricamente, otras nociones como la de origen, causa, medio, agentividad, etc. (Moulton 1963: 302-303 y Bortone 2010: 70 y 2013) ${ }^{11}$.

Es necesario recordar que en época helenística ỏ $\pi$ ó ha invadido el dominio de otras preposiciones ${ }^{12}$ como $\dot{\kappa} \kappa, \pi \alpha \rho \alpha ́$ y, sobre todo, el del fonológicamente similar vंđó, por ejemplo, en expresiones causales. Además, sustituye parcialmente al genitivo de separación, aunque el uso de este último también se encuentra en el NT (Moulton 1963: 302-303 y Wallace 1996: 368). Así, el significado y uso de ả el NT se expande nocionalmente de una manera nueva, mostrando significados atestiguados en época clásica solo rara vez, pero que en la koiné se dan con una frecuencia sin precedentes (Bortone 2010: 185).

\section{II.1. La expresión de la causa mediante à $\pi$ ó + genitivo (en lugar de $\dot{\pi} \boldsymbol{x}$ ) en el NT}

El uso de la preposición àđó en lugar de viđó está atestiguada en época helenística como expresión de causa (Luraghi 1996a : 106); dicho significado se desarrolló, a partir del valor local del morfolexema, mediante la denominada metáfora de fuente indicando el punto en el espacio desde el que se origina la acción verbal. No obstante, la diferencia en el uso de ambas preposiciones, cuando puede percibirse, consiste en

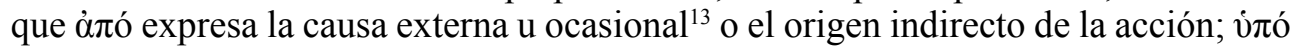
por el contrario, indica la causa interna o eficiente ${ }^{14}$, así como el origen directo de una acción (Harris 2012: 222).

Del mismo modo, aunque la causalidad prototípica es representada principalmente por nombres abstractos, especialmente, de acción o estado ${ }^{15}$ (o pronombres cuyos referentes son sustantivos de esta naturaleza), y se codifica mediante un sintagma

11 En primer lugar, casi todas las preposiciones desplegaron un significado temporal que, en algunos casos, apareció ya en Homero. Además de tiempo, el sentido de las adposiciones espaciales alcanza dominios más abstractos, como la causalidad. Este fenómeno se limita, entonces, a surgir cuando algunas preposiciones rigen sustantivos abstractos, lo que da lugar a una interpretación abstracta de la relación denotada por estas, es decir, la expansión semántica depende todavía del significado léxico. Más tarde, una vez que el nuevo desarrollo semántico se ha consumado, gramaticalizándose, aparecen sustantivos concretos para denotar causa o propósito (Luraghi 2006: 495-496).

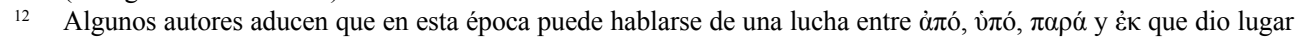
a la progresiva desaparición de las tres últimas en favor de ỏ ó (Harris 2012: 57-58). Así, en esta etapa de la

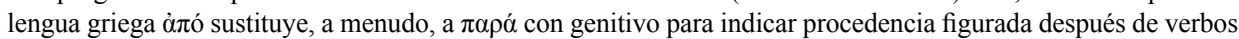
de posesión, recepción o aprendizaje como $\pi \alpha \rho \alpha \lambda \alpha \mu \beta \alpha ́ v \omega, \mu \alpha v \theta \alpha ́ v \omega$, $\alpha \kappa o v ́ \omega$ (Moulton 1963: 258-259). Ello es debido al histórico aumento de la sinonimia en el sistema preposicional griego, lo que dio lugar a un frecuente solapamiento en el uso de algunos de estos morfolexemas característico del griego helenístico, aunque algunas de estas preposiciones eran ya sinónimas en griego clásico (Bortone 2010: 67 y 165). Finalmente, algunas de ellas se volvieron prescindibles y cayeron en desuso. Es reseñable, además, que muchas adposiciones manifiestan sinonimia especialmente en los sentidos no espaciales. Es más, aquellas que poseen un sentido espacial semejante despliegan a menudo significados abstractos similares o idénticos (Bortone 2010: 147-148 y 150).

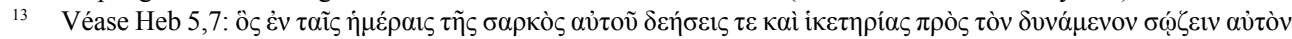

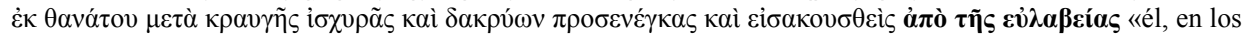
días de su vida mortal, ofreció oraciones y súplicas, a gritos y con lágrimas, al que podía salvarlo de la muerte, y Dios lo escuchó a causa de su piedad».

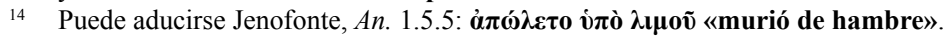

15 El influjo de los rasgos semánticos sobre el uso sintáctico de las categorías léxicas ha sido reconocido en repetidas ocasiones por los estudios lingüísticos de los últimos veinte años. Dichas características semánticas corresponden en la realidad a atributos propios de determinados referentes, según los cuales un cierto rasgo semántico determina la aparición de un lexema en un sintagma determinado, a tenor de nuestras creencias sobre el mundo y la estructura de los eventos. 
preposicional, esta puede asignarse a una extensa variedad de realidades, ya que no existe una clase natural de causas, pues estas pueden ser fuerzas naturales, emociones, conceptos abstractos, seres animados y, a menudo, sucesos. Así, por ejemplo, el prototipo de causa suele convertirse en expresión del causante mediante una simple metonimia de la parte por el todo ${ }^{16}$. Todo ello dificulta, en consecuencia, la especificación de los rasgos léxicos propios de los términos con los que la preposición puede articular este valor. Además, el valor que nos ocupa puede aparecer en una amplia diversidad de acciones (Luraghi 1994: 236 y 1996 : 140 y Martínez Vázquez 2000ª: 646-647).

La semántica funcional define, prototípicamente, la función causa ${ }^{17}$ como una «entidad, situación o persona que provoca o posibilita un estado de cosas sin tener control sobre él», ya que los rasgos característicos de la causa son, únicamente, cierto grado de intencionalidad y control (Martínez Vázquez 2000ª : 640-641). Igualmente, en aquellos casos en los que se atribuye la función causal a seres humanos, lo que es habitual, estos suelen provocar la realización de la acción de manera indirecta y, generalmente, sin intención ni control sobre esta (Luraghi 1994: 236 y 1996 ${ }^{\text {b }}$. 117,122 y 144). Además, se diferencia del agente de predicación pasiva en que la causa no necesita concurrir con otra entidad que soporte un cambio de estado (un paciente), (Luraghi 2013 ${ }^{\mathrm{a}}$.

\section{II.2. La expresión del agente mediante à $\pi$ ó + genitivo (en lugar de í $\pi$ ) en el NT}

Es destacable que el empleo de ảjó en lugar v́đó como medio gramaticalizado de articulación del agente de la predicación pasiva en el NT, atestiguado también en época helenística, es marginal y aparece, sobre todo, en construcciones que emplean verbos de semántica ablativa (especialmente con dicha preposición como preverbio) o de escasa transitividad y con agentes que no son prototípicamente humanos (Luraghi 1996a: 106; 2000: 288 y 2013 ${ }^{\mathrm{a}}{ }^{18}$. Nos encontramos, por tanto, ante un desarrollo

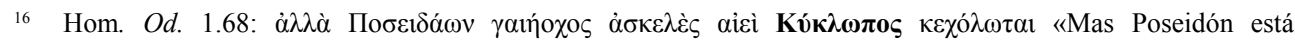
constantemente encolerizado por (el asunto de) el Cíclope».

17 Conti (1999: 229-301) divide la causa en inanimada y animada o causante. De este último existen dos variantes, causante involuntario y responsable. El causante involuntario es el individuo que desencadena el estado de cosas descrito en la oración mediante una situación que escapa a su control. El responsable es la persona que provoca el estado de asuntos que presenta la oración mediante una acción controlada. Este último puede actuar, a su vez, con o sin intencionalidad. Así, el responsable sin intencionalidad es el sujeto que suscita cierto estado de cosas mediante una acción controlada, aunque no intencionada. Por último, el responsable con intencionalidad es el individuo que ocasiona el estado de cosas por medio de una acción controlada e intencionada. Cabe objetar, como observa, Martínez Vázquez (2000a: 644) que la oposición entre el responsable con intencionalidad y el prototipo de agente con frecuencia es inapreciable.

Igualmente, es posible diferenciar entre causa subjetiva o motivo (también denominada causa psicológica o causa interna) y causa objetiva o externa. La primera denota la razón por la que un agente lleva a cabo una acción determinada y, generalmente, designa un concepto abstracto o un suceso. Así, la característica principal de la razón es que aparece en confluencia con un agente y solo se produce en acciones controladas. Un ejemplo

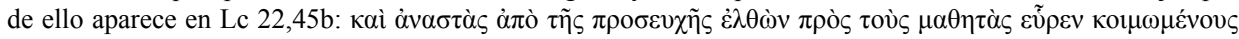

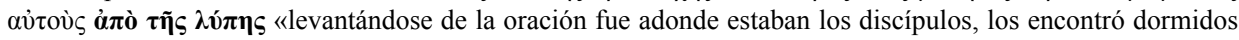
por la tristeza». La causa objetiva es atribuida a una entidad, concepto abstracto o suceso que provoca o hace

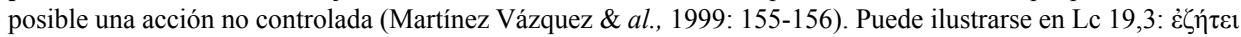

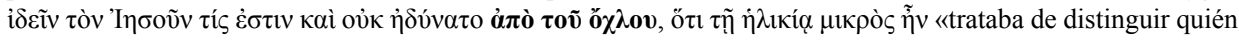
era Jesús, pero no podía debido a la gente / pero la gente se lo impedía».

18 Un ejemplo de ello es el siguiente pasaje de Lucas donde la preposición aparece regida por un verbo de semántica

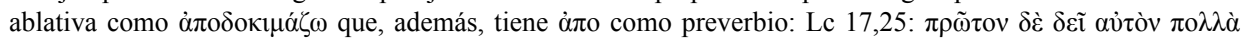


limitado que se da en condiciones gramaticales particulares (Coulter 2005: 246 y $262)^{19}$. Sin embargo, la diferencia entre el empleo de ambos morfolexemas radica (cuando puede apreciarse) ${ }^{20}$, en que ả ${ }^{2}$ denota una agentividad más remota o menos activa que $\dot{v} \pi \mathrm{o}^{21}$ y esta, por el contrario, indica la agentividad inmediata ${ }^{22}$.

El valor agentivo de à $\pi$ se desarrolló también, según Luraghi (2000: 282-285), a partir de su valor local, mediante la denominada metáfora de fuente. Sin embargo, la multiplicidad de codificaciones alternativas que sirvieron para expresar la función agentiva a lo largo de la historia de la lengua griega ${ }^{23}$ supone, según algunos autores, una evidencia que constata la interpretación del agente de la predicación pasiva como variante conceptual de la noción de causa en griego ${ }^{24}$. Así, tanto el citado sintagma locativo vं $\pi$ ó + dativo (genitivo en Homero) como algunas expresiones

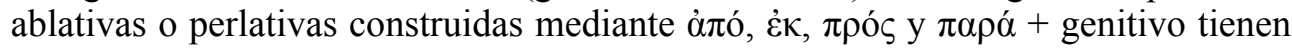
en común que, además de articular el agente de la voz pasiva, fueron expresión de causa (Coulter 2005: 193).

La función agente es definida por la semántica funcional como la «entidad, típicamente un ser consciente, que controla de modo activo, consciente y voluntario la acción» (Martínez Vázquez 2000a: 640-641). Dicho valor suele aparecer en acciones altamente transitivas que cuentan con un paciente, cuyo rasgo básico es la afectación, ya que se somete a un cambio de estado que es efecto de la acción verbal (Luraghi 2013 ${ }^{\mathrm{a}}$ ). Igualmente, entre sus características se encuentra la de ser un ser animado y consciente, así como la intencionalidad, el control (posee el dominio sobre la acción, esto es, decide llevarla a cabo y es responsable de su realización) y la ejecución (realiza la acción, interviniendo directamente en ella).


por esta generación».

19 En efecto, aunque su aparición es temprana en los LXX y en el NT, átó no fue la preposición dominante para la codificación del agente de la voz pasiva ni en los papiros de la antigüedad tardía ni en algunos textos del período bizantino ni, por último, en los textos de la antigüedad clásica. Otras preposiciones de significado ablativo o

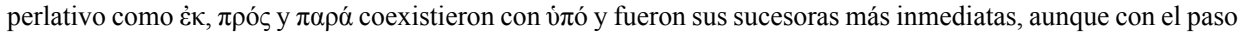
del tiempo, algunas de ellas cayeron en desuso; de hecho, el uso agentivo de ảjó no ocupó su lugar preeminente hasta después del comienzo del s. XII (Coulter 2005: 222).

20 Zerwick observa que, a menudo, no podemos deducir sutiles diferencias del empleo de una por otra en el NT (Zerwick 1979: 54).

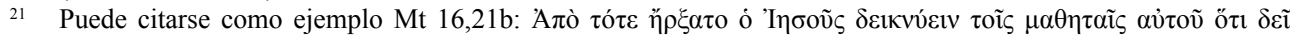

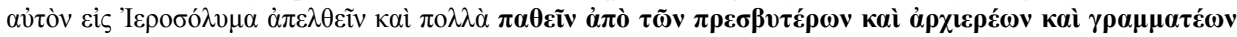

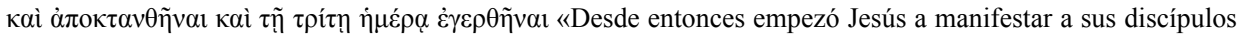
que tenía que ir a Jerusalén, padecer mucho a manos de los senadores, sumos sacerdotes y letrados, ser ejecutado y resucitar al tercer día». El valor de la preposición en este pasaje será estudiado en profundidad en las siguientes páginas.

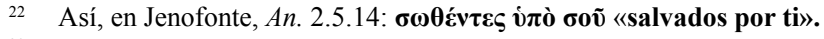

23 Véase nota 19.

24 Esta observación puede ser completada, según la semántica cognitiva, atendiendo a la perspectiva como un elemento determinante de la semántica de las unidades lingüísticas, no solo para la caracterización del prototipo de una función semántica, sino para establecer la escala de marginalidad de las variantes nocionales que conforman el haz conceptual de dicho valor, así como las vías de confluencia con otras categorías dentro del continuum agente-causa-intermediario (Martínez Vázquez 2000: 656). Así, al igual que el agente de predicaciones pasivas, la función causal prototípica nunca es sujeto de la predicación ni un complemento valente (o argumento, según la gramática funcional-cognitiva), sino un elemento no valencial, es decir, un circunstancial o satélite. En consecuencia, se trata de una situación o entidad que suscita un hecho dado desde la periferia de la escena nocional o, lo que es lo mismo, desde un plano secundario (según el punto de vista de quien imagina el hecho), sin incidir directamente en él (Martínez Vázquez 2000`: 648-649). Por ello, debe alegarse que todo agente de la frase pasiva es una variedad de la noción de causa, ya que lo que lo caracteriza es su naturaleza adyacente (Martínez Vázquez 2000a 650 y 656). 


\section{El continuum conceptual agente-causa-intermediario}

A lo dicho anteriormente, hay que añadir que el influjo de la gramática cognitiva ha hecho prevalecer en los últimos años la idea de que las funciones semánticas no constituyen parcelas conceptuales nítidamente separadas, sino que son, más bien, nociones graduales que tienen algunos puntos de estrecha proximidad y otros de máxima diferenciación. De este modo, algunos de los roles semánticos relacionados más estrechamente con la causalidad, como el valor de agente (de predicación pasiva), serían definidos como categorías radiales estructuradas en torno a un prototipo que suele caracterizarse por una serie de propiedades (en el caso de la causa, la ausencia, total o parcial, de intencionalidad y control y, en el caso del agente, la animación, la intencionalidad, el control y la ejecución).

Así, dichos rasgos no determinan por sí solos, de forma aislada, la pertenencia a la categoría, ya que cualquiera de estas características puede faltar y, por tanto, dicho elemento podrá ser considerado miembro no prototípico de la categoría (Luraghi 1989: 296 y 302, y Martínez Vázquez 2000ㄹ 486-488). En consecuencia, algunas de las propiedades citadas, como el rasgo de control o el de ejecución pueden oscilar en la representación del rol de agente y causa dentro del continuum agente-causaintermediario ${ }^{25}$.

\section{Algunos casos de controversia respecto al valor de la preposición ḋ $\pi$ ó en el NT como marcador de causa o agente}

La consulta del lema correspondiente a ả $\pi$ ó en los diccionarios $L S J\left(1996^{15}\right.$, s.v.

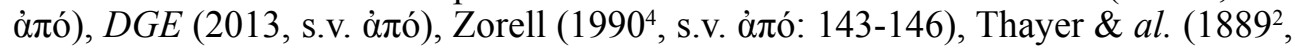
s.v. $\dot{\alpha} \pi$ ó), $B D A G\left(2000^{3}\right.$, s.v. $\dot{\alpha} \pi$ ó y Louw \& Nida $(1988$, s.v. $\dot{\alpha} \pi$ ó) revela frecuentes contextos en los que existe desacuerdo entre estos léxicos acerca del valor del morfolexema, especialmente, cuando este indica causalidad o agentividad. La misma división de opiniones queda corroborada, como veremos, con la lectura de diferentes traducciones bíblicas elegidas al azar como la New Revised Standar Version (NRSV),

25 Por tanto, una cualidad tan característica del agente estándar como la de ejecución de la acción, no resulta del todo decisiva, ya que a menudo se caracteriza al participante menos directo en la acción como agente y al más inmediato como intermediario. Por ello, en estos casos, será el rasgo de control el que caracterizará al agente, ya que es él quien ejerce el control sobre la acción, aunque no la lleva a cabo; la ejecución recae

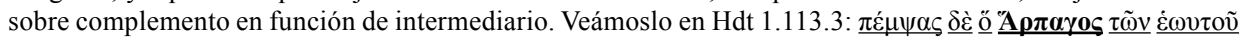

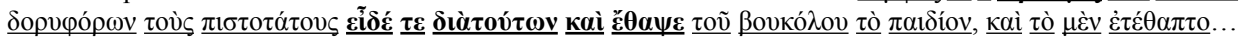
«Harpago envió a sus más fieles lanceros y por medio de ellos vio y sepultó al hijo del pastor. Lo sepultaron y...». Asimismo, la cualidad de ejecución de la acción determinará el rol de agente en aquellos casos en que existiendo un controlador y un ejecutor diferentes, el sujeto es agente que realiza la acción, pero carece de control sobre ella; el control se atribuye, en este caso, al sintagma con función de causa (Martínez Vázquez

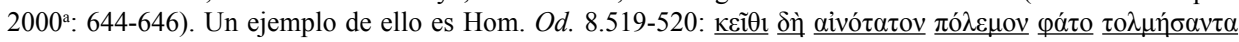

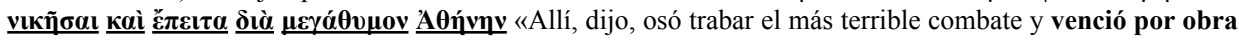
de la magnánima Atenea».

De este modo, la noción de control representa un área de confluencia entre el concepto de agente y el de causa, y constituye el elemento diferenciador entre el agente y el intermediario (Luraghi 1994: 230; 1996 ${ }^{\text {b }}$ 140-141 y Martínez Vázquez 2000ª : 644-646). Por último, es reseñable que la ausencia de control afecta en mayor medida a la identificación del agente que la ausencia de intencionalidad, debido a que hay acciones controladas que pueden llevarse a cabo de forma voluntaria o involuntaria (Martínez Vázquez 2000 $: 493$ ). Por su parte, la idea de ejecutor se encuentra en la confluencia entre la noción de agente y la de intermediario, y distingue al agente de la causa. 
(1989), King James Version (KJV), (1982), la de Reina Valera (RV), (2010), la de Nácar Colunga (NC), (1999) y la de Cantera Iglesias (CI), (2015).

Así, de acuerdo con los resultados del examen de la preposición que nos ocupa por parte del DGENT, especialmente, para el valor causal y agentivo, así como con los rasgos semánticos propios de dichas nociones establecidos por la semántica funcional y cognitiva, trataremos de dilucidar el sentido de ả $\pi$ ó en cuatro pasajes del NT donde el uso de la preposición es valioso para la interpretación del pasaje a un nivel exegético. Se demostrará que tanto los significados como las traducciones ofrecidas para la preposición en dichos versículos por parte de los léxicos y las traducciones citadas distorsionan, con frecuencia, su comprensión, debido a la ausencia de rigor metodológico y a la falta de atención al contexto en que aparece el morfolexema. Para ello, examinaremos el morfolexema, en primer lugar, centrándonos en su vinculación al contexto de acuerdo con la metodología del $D G E N T$ y, en segundo lugar, comentaremos cada uno de estos pasajes, comparando nuestra traducción ${ }^{26}$ con el resto de versiones citadas, así como con el sentido y la traducción correspondiente ofrecida por los citados diccionarios, y por algunos estudios y monografías específicas.

\section{IV.1. Le 16,18 y Mt 16,21b. à ó por vं $\pi$ ó}

Lc 16,18

El evangelio de Lucas ofrece un caso de controversia tanto entre los diccionarios y estudios específicos como entre las traducciones respecto al significado y la tra-

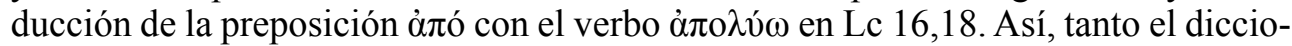
nario $B D A G\left(2000^{3}\right.$, s.v. $\dot{\alpha} \pi$ ó $)$ como el $D G E(2013$, s.v. $\alpha \dot{\alpha}$ ó $)$ consideran que la adposición denota separación. En cambio, Coulter (2005: 242) en su estudio sobre la agentividad en griego antiguo ya cita este texto como uno de los frecuentes casos en los que el morfolexema aparece como marcador de agente de un verbo en voz pasiva.

En cuanto a las distintas traducciones, la NRSV (1989) y la KJV (1982) atribuyen a la adposición en este versículo el valor de marcador de separación:

NRSV (1989): «Anyone who divorces his wife and marries another commits adultery, and whoever marries a woman divorced from her husband commits adultery».

KJV (1982): «Whosoever putteth away his wife, and marrieth another, committeth adultery: and whosoever marrieth her that is put away from her husband committeth adultery».

Dichas versiones contrastan, sin embargo, con las de CI (2015) y NC (1999), las cuales presentan la preposición articulando el rol de agente de predicación pasiva

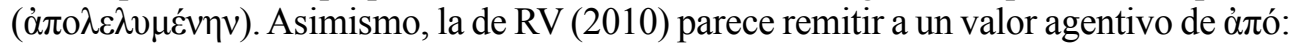

CI (2015): «Todo el que repudia a su mujer y se casa con otra comete adulterio; y el que se casa con una que ha sido repudiada por su marido, comete adulterio». NC (1999): «Todo el que repudia a su mujer y se casa con otra, adultera, y el que

26 Como versión de referencia para este trabajo hemos tomado la de Mateos-Schökel ( ${ }^{32001) .}$ 
se casa con la repudiada por el marido, comete adulterio».

RV (2010): «Todo el que repudia a su mujer y se casa con otra, adultera; y el que se casa con la repudiada del marido, adultera».

La idea de separación que emana del verbo ỏ $\pi$ $\lambda \hat{\omega} \omega$ 'liberar', 'poner en libertad', 'soltar', 'desatar' parece ser, en nuestra opinión, el origen de la dificultad en la interpretación del valor del morfolexema preposicional que nos ocupa, la cual puede resolverse atendiendo, principalmente, al contexto. Es cierto que el sentido local dinámico de separación de ảđó según el análisis del DGENT (2007 s.v. àđó: 789829), surge cuando la preposición aparece, entre otros, con verbos de rehabilitación, eliminación o liberación, como en este caso $(\dot{\alpha} \pi \circ \lambda \hat{v} \omega)$. Sin embargo, puede objetarse que la codificación del morfolexema como marcador no estándar de agente de

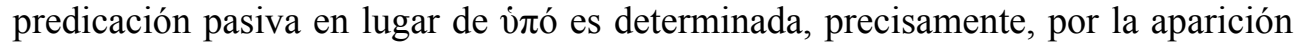
de verbos de semántica ablativa en voz pasiva con ḋंó como preverbio, como $\alpha \dot{\pi} 0 \lambda v ́ \omega$. A ello hay que añadir que, en cuanto al aspecto sintáctico, suelen estar en forma participial (Coulter 2005: 113-115 y 246), lo cual también se cumple aquí

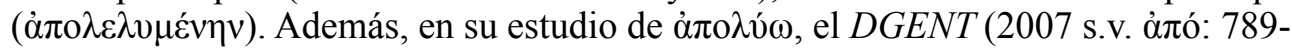
829) explica que cuando este aparece con acusativo de persona femenino adquiere sentido jurídico y denota el acto de liberar a la mujer del vínculo matrimonial, traduciéndose, en ese caso, por 'repudiar' ${ }^{27}$. De este modo, hay que destacar que, según la concepción veterotestamentaria, era únicamente el marido quien podía repudiar a la mujer y contraer nuevas nupcias, pero no a la inversa, lo que queda explicitado por el uso del morfolexema como marcador de agente (la prohibición de repudiar a la mujer y volverse a casar está dirigida al hombre; obsérvese que tanto los sujetos como sus correspondientes participios aparecen en forma masculina),

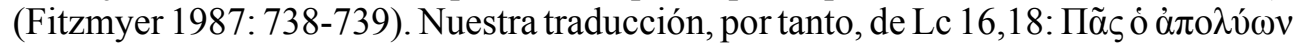

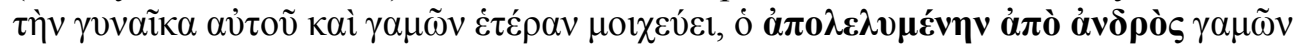
$\mu o \imath \chi \varepsilon v ́ \varepsilon 1$ «Todo el que repudia a su mujer y se casa con otra, comete adulterio; y el que se casa con una repudiada por su marido comete adulterio», parece más fiel a la mentalidad del judaísmo de la época, la cual queda reflejada por las prácticas habituales a las que alude Lucas en este pasaje y contra las que Jesús establece como norma la monogamia, situando al hombre y la mujer en el mismo nivel respecto al adulterio (Green 1997: 603).

Por lo demás, nos encontramos ante un agente estándar, ya que cuenta con todos los rasgos propios de esta categoría (animación, voluntariedad, control y ejecución), por lo que, como se dijo anteriormente, tal vez este sea uno de esos casos en los que no podemos deducir sutiles diferencias del empleo de àđó por vं $\pi$ ó.

\section{Mt $16,21 \mathrm{~b}$}

El significado de la preposición àđó en este pasaje de Mateo es también objeto de diferentes interpretaciones entre los diccionarios, estudios y gramáticas. Así, el léxico de Thayer $\left(1889^{2}\right.$, s.v. àmó) aduce el citado versículo como ejemplo del valor causal de la preposición. Sin embargo, tanto el diccionario de Zorell $\left(1990^{4}\right.$, s.v. àtó: $143-146)$ y el de $B D A G\left(2000^{3}\right.$, s.v. $\alpha$ ó $)$ como Robertson (1914: 579-580) y Harris (2012: 58-59) consideran que à $\pi$ á aparece aquí en lugar vं $\pi$ ó como codificador de

27 El $D G E(2013$, s.v. $\alpha$ đó) llega a la misma conclusión en su examen de $\dot{\alpha} \pi$ o $\lambda v ́ \omega$. 
agente de la voz pasiva, pudiendo traducirse por 'a manos de' 'por orden de' e incluso 'por parte de' (at the hands of, by command of), traducciones que reflejan la noción de agentividad indirecta propia del morfolexema. En cuanto a las diferentes versiones de este pasaje, todas parecen decantarse también por una traducción de áxó como marca de agente, algunas, como en el caso de la versión de la NRSV (1989) y la de RV (2010), traduciendo por 'a manos de', y otras, como la de NC (1999), CI (2015) y KJV (1982), por 'por parte de':

NRSV (1989): «From that time on, Jesus began to show his disciples that he must go to Jerusalem and undergo great suffering at the hands of the elders and chief priests and scribes, and be killed, and on the third day be raised».

RV (2010): «Desde entonces comenzó Jesús a declarar a sus discípulos que le era necesario ir a Jerusalén y padecer mucho a manos de los ancianos, de los principales sacerdotes y de los escribas, y ser muerto, y resucitar al tercer día». NC (1999): «Desde entonces comenzó Jesús a manifestar a sus discípulos que tenía que ir a Jerusalén para sufrir mucho de parte de los ancianos, los príncipes de los sacerdotes y los escribas, y ser muerto, y al tercer día resucitar».

CI (2015): «Desde entonces Jesús empezó a declarar a sus discípulos que él tenía que ir a Jerusalén y sufrir mucho de parte de los ancianos y sumos sacerdotes y escribas, y sufrir la muerte, y al tercer día resucitar».

KJV (1982): «From that time forth began Jesus to shew unto his disciples, how that he must go unto Jerusalem, and suffer many things of the elders and chief priests and scribes, and be killed, and be raised again the third day».

La consideración por parte de Thayer de la función del morfolexema en este contexto como causa eficiente no resulta del todo convincente debido a que el pasaje refleja un antiguo uso de la preposición vं ó + genitivo junto al verbo $\pi \alpha ́ \sigma \chi \omega$ 'ser bien o mal tratado (por alguien)', 'sufrir a manos de (alguien)', (1996 ${ }^{15}$ [2005, s.v. ò Por tanto, en esta construcción, el verbo adquiere sentido pasivo ${ }^{28}$ y el sintagma preposicional regido por áxó codifica el complemento agente de la frase. En consecuencia, nos encontramos ante un caso de sustitución de áđó por víó. Además, su aparición junto a un verbo de baja transitividad como $\pi \alpha ́ \sigma \chi \omega$, constituye otro rasgo determinante de la articulación del agente (indirecto) de predicación pasiva por parte del morfolexema que nos ocupa (Luraghi 2013 a).

Desde el punto de vista semasiológico, los rasgos que caracterizan al agente en este contexto son los de animación, voluntariedad, control y ejecución, si bien es cierto que la agentividad claramente indirecta que articula la preposición en este pasaje aproxima el valor del morfolexema en este versículo a la noción de causa. El valor de la adposición en este pasaje es destacable en términos exegéticos, ya que los sumos sacerdotes y los letrados, todos ellos miembros del Gran Consejo (Mateos 1987: 112-113), es decir, los tres órdenes constitutivos del Sanedrín, quedan representados como los responsables (agentes indirectos) del sufrimiento de Jesús, pues estos son sus primeros perseguidores, quienes decidirán en nombre de la nación que Jesús debe morir, persuadiendo a la gente para que apoye la sentencia, aunque, propiamente, fue el poder romano el agente ejecutor de su condena y de

28 Es necesario recordar que originalmente $\pi \alpha ́ \sigma \chi \omega$ expresaba un estado pasivo (Chantraine 19774: 878). 
su muerte ${ }^{29}$. Así queda sugerido por la versión de este pasaje que proponemos:

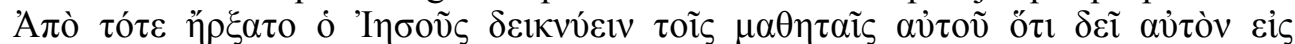

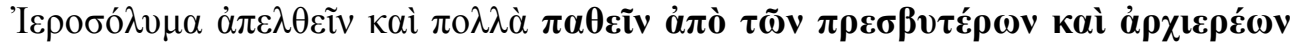

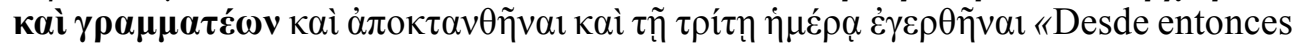
empezó Jesús a manifestar a sus discípulos que tenía que ir a Jerusalén, padecer mucho a manos de los senadores, sumos sacerdotes y letrados, ser ejecutado y resucitar al tercer día».

En suma, puede deducirse que, en este contexto, el morfolexema sustituye a vió en la codificación de la noción de agente de la acción verbal, lo que queda, también, manifestado por la acorde traducción con este valor por parte de las diferentes versiones citadas.

\section{IV.2. 1Cor 11,23. ’̉ $\pi$ ó por $\pi \alpha \rho \alpha ́$}

Algunos diccionarios parecen contradecir unánimemente las diferentes versiones elegidas en cuanto al significado de la preposición en 1Cor 11,23. De este modo,

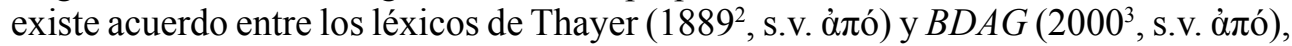
acerca del sentido de ảió en este pasaje, ya que ambos lo consideran un ejemplo del valor de la preposición como codificador de causa eficiente referida a personas.

En cuanto a las distintas versiones neotestamentarias elegidas, estas traducen la preposición con valor de origen o procedencia:

NC (2015): «Porque yo he recibido del Señor lo que os he transmitido, que el Señor Jesús, en la noche en que fue entregado, tomó el pan...».

CI (1999): «Pues yo recibí del Señor lo que a mi vez os transmití: que el Señor Jesús, la noche en que era entregado, cogió pan...».

KJV (1982): «For I have received of the Lord that which also I delivered unto you, That the Lord Jesus the same night in which he was betrayed took bread...». NRSV (1989): «For I received from the Lord what I also handed on to you, that the Lord Jesus on the night when he was betrayed took a loaf of bread...».

RV (2010): "Yo recibí del Señor lo que también os he enseñado: Que el Señor Jesús, la noche que fue entregado, tomó pan...».

Para elucidar cuál de los dos valores puede atribuirse a la preposición en este pasaje referido a la transmisión de la tradición eucarística, es necesario, nuevamente, prestar atención al contexto. El posible significado causal del morfolexema en este pasaje puede refutarse reparando en el verbo que rige la adposición y que, en este caso, es $\pi \alpha \rho \alpha \lambda \alpha \mu \beta \alpha ́ v \omega$ 'tomar consigo'; 'recibir', 'heredar'; el estudio del

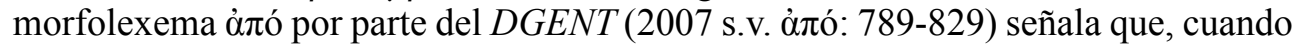
la preposición aparece, entre otros, con verbos de posesión, recepción o aprendizaje, indicando la persona o el colectivo de quien se requiere o recibe algo, à $\pi$ ó adquiere valor de origen o procedencia. Esta conclusión es precisada tanto por Moulton (1963: 258-261) como por Zerwick (2008: 610) añadiendo que se trata de un caso en el que ả $\alpha$ ó sustituye a la preposición $\pi \alpha \rho \alpha ́$. Este dato es relevante también desde un punto de vista exegético, ya que, en este caso, $\alpha \pi$ ex expresa junto a $\pi \alpha \rho \alpha \lambda \alpha \mu \beta \alpha ́ v \omega$ una transmisión más indirecta o una mayor distancia de la fuente de procedencia

29 Bermejo Rubio ha profundizado en esta cuestión en un estudio reciente (2016: 528-530). 


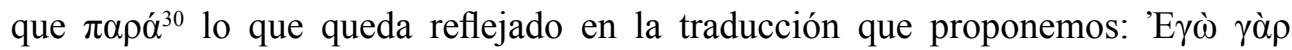

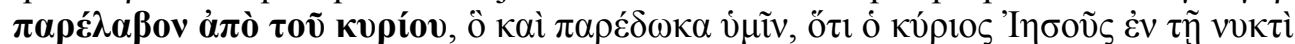

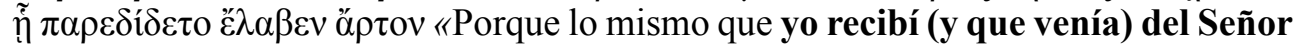
os lo transmití a vosotros: que el Señor Jesús, la noche en que iban a entregarlo, cogió un pan». De este modo, aunque no podemos saber cómo recibió Pablo la antigua tradición cristiana de la cena eucarística, este uso intencionado de ả $\pi_{0}$ por $\pi \alpha \rho \alpha ́$ podría sugerir que este no la aprendió de manera directa mediante una revelación, como señalan algunos estudiosos, sino, de manera menos inmediata, por tradición oral (a la que apunta $\pi \alpha \rho \alpha \lambda \alpha \mu \beta \alpha ́ v \omega$, según Zerwick 2008: 610) de alguno de los doce. De otro modo, se hubiera preferido el uso de $\pi \alpha \rho \alpha ́$. En suma, el examen de los datos aportados parece confirmar que el morfolexema indica aquí procedencia u origen, no causalidad. Puede cotejarse, además, el mismo error en otros versículos del $\mathrm{NT}^{31}$. Un último dato que parece corroborar la correcta interpretación del morfolexema en el pasaje elegido como marcador de procedencia u origen es la unánime traducción con este valor por parte de las diferentes versiones citadas.

\section{IV.3. Le 12,57}

Cierto desacuerdo entre algunos diccionarios y traducciones en torno al sentido de la preposición ò $\pi_{\text {ó }}$ puede observarse a tenor de su análisis del morfolexema en Lc 12,57. En cuanto a las distintas versiones del NT seleccionadas, tanto la de CI (2015) como la de NC (1999), la RV (2010) y la NRSV (1989) muestran una traducción similar del morfolexema: las biblias castellanas traducen 'por', mientras que las inglesas traducen 'for', lo cual hace pensar, indistintamente, en una posible interpretación agentiva, causal o, tal vez, de intermediación del morfolexema en este versículo:

CI (2015): «¿Y por qué no distinguís por vosotros mismos lo que es justo?».

NC (1999): «¿Por qué no juzgáis por vosotros mismos lo que es justo?».

RV (2010): «¿Por qué no juzgáis por vosotros mismos lo que es justo?».

NRSV (1989): «And why do you not judge for yourselves what is right?».

La King James Version (KJV), (1982), por su parte, parece apuntar a un valor de procedencia u origen, traduciéndolo por 'of':

KJV (1982): «Yea, and why even of yourselves judge ye not what is right?».

30 Harris (2012: 63-64) afirma, sin embargo, que el uso de $\alpha$ ó en lugar de $\pi \alpha \rho \alpha ́$ en este versículo puede deberse a un simple cambio estilístico para evitar un triple uso $\pi \alpha \rho \alpha ́$ en el mismo pasaje (no solo aparece como preverbio

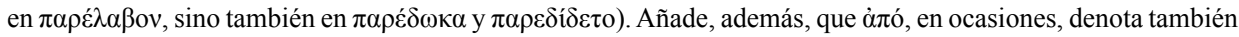
relaciones no mediadas, como en Mt 11,29; Col 1,7; 1Jn 1,5). Robertson (1914: 578-579) señala que, aunque no son exactamente sinónimas, no debe exagerarse la diferencia entre una y otra.

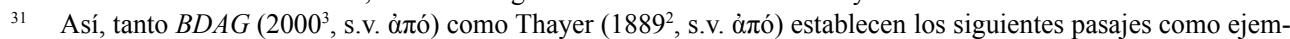
plos del valor de la preposición como codificador de causa eficiente referida a personas: Mt 12,38; Mt 17,25ab; Mt 17,26; Lc 22,71; Hch 9,13; Rom 1,7; 1Cor 1,30; 1Cor 4,5; Ef 6,23; Flp 1,2; 1Jn 1,5; 1Jn 2,20; 1Jn 2,27; 1Jn 3,22; 1Jn 4,21; Ap 1,4. También en fórmulas de saludo como en 1Cor 1,3; 2Cor 1,2; Gál 1,3; Ef 1,2; Col 1,2; 2Tes 1,2; 1 Tim 1,2; Tit 1,4; Flm 3. No obstante, según el DGENT (2007 s.v. à denota en todos estos casos origen o procedencia, ya que el verbo que rige la preposición suele ser de, recepción, deseo o posesión, factor contextual que determina este valor. 
Por lo demás, el diccionario $B D A G\left(2000^{3}\right.$, s.v. àđó $)$ señala que el sintagma $\dot{\alpha} \varphi^{\prime} \dot{\varepsilon} \alpha v \tau \tilde{\omega} v$ refleja un uso agentivo de la preposición bastante común. El léxico de Thayer $\left(1889^{2}\right.$, s.v. $\dot{\alpha} \pi$ ó), en cambio, aduce un valor causal del morfolexema en este contexto, indicando, asimismo, que es una expresión especialmente común en Juan.

Esta disparidad de opiniones en torno a la noción que expresa aquí la preposición puede resolverse atendiendo nuevamente al contexto. En primer lugar, es necesario

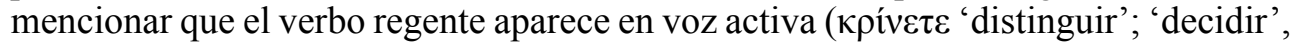
'juzgar'), lo que descarta un posible uso del morfolexema como marcador de agente de predicación pasiva. Es destacable, sin embargo, desde un punto de vista semántico, que los rasgos de control y ejecución (además de animación y voluntariedad) aparecen tanto en el núcleo del sintagma preposicional $\alpha \dot{\varphi} \varphi$ غ $\dot{\varepsilon} \alpha \tau \tilde{\omega} v^{32}$, que es co-referencial respecto al sujeto agente, como en este, el cual está implícito en este contexto; sin embargo, $\alpha \varphi^{\prime} \dot{\varepsilon} \alpha v \tau \tilde{\omega} v$, enfatiza el rasgo de ejecución de la acción, atenuándolo en el agente elidido. Del mismo modo, los rasgos de voluntariedad y control quedan mitigados en $\dot{\alpha} \varphi$ ' $\dot{\varepsilon} \alpha v \tau \tilde{\omega} \nu$ y enfatizados en el agente, ya que este rasgo es lo que diferencia típicamente al agente del intermediario. Puede añadirse, además, que esta acepción aparece en griego no solo con verbos de acción o proceso, sino también de lengua y sentido ( $D G E 2013$, s.v. $\dot{\alpha} \pi$ ó). Por todo ello, el morfolexema puede considerarse aquí codificador de intermediación denotando la persona que lleva a cabo la acción en nombre del agente. El valor causal alegado por el diccionario de Thayer $\left(1889^{2}\right.$, s.v. $\dot{\alpha} \pi$ ó puede descartarse argumentando que la causa no interviene, por lo general, directamente en la acción, es decir, un causante carecería del rasgo de ejecución.

El valor de intermediación es acorde con el sentido del pasaje, ya que Jesús exhorta a la gente a independizarse de los fariseos, alegando que no necesitan su doctrina como medio para saber lo que es bueno o malo y animándola a «valerse de sí misma» ( $\dot{\alpha} \varphi$ ' $\dot{\varepsilon} \alpha v \tau \tilde{\omega} v)$ para enjuiciar y hacer lo que convenga en cada circunstancia

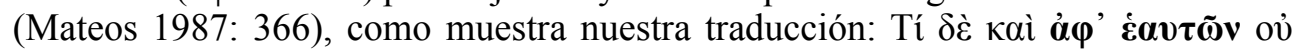

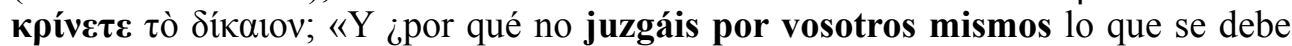
hacer?». El mismo peculiar desdoblamiento del sujeto en agente e intermediario puede observarse en otros pasajes del $\mathrm{NT}^{33}$.

\section{Conclusiones}

Como se ha podido constatar, para abordar el estudio de los valores de esta clase de morfolexemas es necesario partir de un enfoque metodológico principalmente semántico (sin olvidar el aspecto morfosintáctico), así como estructural, pues el contexto constituye un factor indispensable para fijar el sentido de esta clase de morfolexemas. Ello es debido a que el sistema preposicional griego se encuentra a caballo entre lo lexical y lo gramatical, ya que el sentido de esta especie gramatical es el resultado de la combinación del significado de la preposición y del morfema casual con el que esta se vincula, así como del contenido léxico del núcleo del sintagma, del predicado y de otros factores contextuales.

\footnotetext{
3. ${ }^{\mathrm{a}}$ persona en lugar de la 2 . $^{\mathrm{a}}$ persona del plural.

Véase también Lc 21,30; John 5,19,30; 11,51; 14,10; 16,13; 18,34; 2 Cor 3,5.
} 
Asimismo, de la falta de acuerdo entre algunos de los léxicos examinados sobre el sentido de la preposición ámó en Mt 16,21, Lc 12,57, Lc 16,18 y 1Cor 11,23 se deduce su frecuente falta de rigor metodológico y su imprecisión en el estudio de los diferentes contextos en que aparece el morfolexema, de modo que su análisis falsea con frecuencia el valor de $\alpha$ đó y da lugar a interpretaciones erróneas de su sentido, confundiendo erróneamente entre sí o con otras funciones el valor agentivo (de predicación pasiva) y el causal del morfolexema. La misma inexactitud en el análisis del morfolexema puede atribuirse, en ocasiones, a las diferentes versiones neotestamentarias utilizadas $\mathrm{y}$, finalmente, a las gramáticas y estudios consultados.

Del mismo modo, se ha puesto de manifiesto que las observaciones de la gramática funcional-cognitiva, según las cuales, la articulación metafórica de la agentividad (de predicación pasiva) en época helenística mediante ỏ ó en lugar de vió es una variante conceptual de la noción de causa en griego, coinciden con las de la semántica cognitiva, ya que tanto el valor de causa como el de agente (de predicación pasiva) podrían definirse como categorías radiales estructuradas en torno a un prototipo representado por una serie de propiedades, algunas de las cuales, como el rasgo de control o ejecución, fluctúan, en ocasiones, en la caracterización del rol de agente y causa dentro del continuum agente-causa-intermediario, convirtiéndolos en miembros no prototípicos de la categoría.

Por último, la aplicación de los principios metodológicos del DGENT al análisis semántico de la preposición que nos ocupa ha supuesto, en nuestra opinión, un progreso en el estudio de los valores de ảió en el griego del corpus neotestamentario, ya que ha permitido detectar cuál es el ámbito nocional del morfolexema en los versículos estudiados, así como arrojar luz sobre la interpretación dichos pasajes del NT en los que el valor de la preposición es determinante para su exégesis.

\section{Bibliografía}

Alexandre Júnior, Manuel A. (2016), Gramática de Griego clásico y helenístico, Córdoba, El Almendro.

Bauer, Walter \& Danker, Frederick W. $\left(2000^{3}\right), B D A G$, en Bibleworks 7 [CD-ROM], Norfolk, VA 2006 BibleWorks, Software Program.

Bermejo Rubio, Fernando (2016), «Y vendrán los romanos» (Jn 11,48). Sobre la identidad de los responsables del arresto de Jesús el galileo», en Israel M. Gallarte \& Jesús Peláez, In mari via tua. Philological Studies in Honour of Antonio Piñero, Córdoba, El Almendro: 503-530.

Bortone, Pietro (2010), Greek prepositions: From Antiquity to the Present, Oxford, Oxford University Press.

- (2013), «Adpositions (Prepositions)», en Georgios K. Giannakis [ed.], EAGLL, [en línea] (Koninklijke, Brill NV), en http://referenceworks.brillonline.com/entries/ encyclopedia-of-ancient-greek-language-and-linguistics/adpositions-prepositionsCOM_00000007\#d87365637e1848 (12/04/2016, 20:18).

Cantera, Francisco \& Iglesias, Manuel (2015), Sagrada Biblia, Madrid, B.A.C.

Caragounis, Chrys (2004), The Development of Greek and the New Testament, Tübingen, Mohr Siebeck.

Chantraine, Pierre (19774) DELG. Vols. 1-4, Paris, Éditions Klincksieck. 
Conti, Luz (1999), «La expresión de la causa en homero con referentes humanos», Emerita. Vol. 67. N. ${ }^{\circ}$ 2, 295-313, doi:10.3989/emerita.1999.v67.i2.177.

Coulter H., George (2005), Expressions of agency in Ancient Greek, Cambridge, Cambridge University Press.

Fitzmyer, Joseph A. (1987), El Evangelio según Lucas. Traducción y Comentario. Capítulos 8,22-18,14. Vol III. Madrid, Ediciones Cristiandad.

Galichet, Georges (1947), Essai de grammaire psychologique, Paris, PUF.

Green, Joel B. (1997), The Gospel of Luke. The New International Commentary on the New Testament, Grand Rapids, Michigan, Wm. B. Eerdmans Publishing Co.

Harris, Murray J. (2012), Prepositions and Theology in the Greek New Testament, Grand Rapids, Michigan, Zondervan.

Hays, Richard B. (1997), First Corinthians. Interpretation. A Bible Commentary for Teaching and Preaching, John Louisville, Knoss Press.

Liddell, Henry G. \& Scott, Robert \& al. $\left(1996^{15}\right)$, LSJ, Oxford, Oxford University Press [en línea] (University of California), en http://stephanus.tlg.uci.edu/lsj/\#eid=12585\&context $=1$ sj\&action=from-search $(12 / 12 / 2016,09: 00)$.

Louw, Johannes P. \& Nida, Eugene A. (eds.), (1988), Greek-English Lexicon of the New Testament based on Semantic Domains, 2 vols., en Bibleworks 7 [CD-ROM], Norfolk, VA 2006, BibleWorks, Software Program.

Luraghi, Silvia (1989), «Cause and Instrument Expressions in Classical Greek. Remarks on the Use of $\delta$ í in Herodotus and Plato», Mnemosyne. Fourth Series. Vol. 42, Fasc. 3/4: 294-307.

- (1994), "Animate Nouns in Cause Expression», en Bernard Jacquinod (ed.) Cas et prépositions en grec ancien. Contraintes syntaxiques, Saint-Étienne, Publications de 1'Université de Saint-Étienne: 227-237.

- $\left(1996^{\mathrm{a}}\right)$, «Il rapporto fra caso e preposizione all'interno del sintagma preposizionale», en S. Luraghi (ed.), Studi su casi e preposizioni nel greco antico, Milano, Franco Angeli: 59-110.

- $\left(1996^{\mathrm{b}}\right)$, «Determinazioni di Causa e Strumento nel greco classico», en S. Luragui (ed.), Studi su casi e preposizioni nel greco antico. Milano, FrancoAngeli: 111-145.

- (2000), «Spatial Metaphors and Agenthood in Ancient Greek», en M. Ofitsch \& C. Zinko (eds.), Sonderdruck aus 125 Jahre Indogermanistik in Graz, Karl Franzens-Universität Graz, Leykam: 275-290.

- (2003) On the Meaning of Prepositions and Cases. The expression of semantic roles in Ancient Greek (Amsterdam-Philadelphia PA), en http://books.google.es/books?id=tULh yKKX9nsC\&printsec $=$ frontcover\&hl $=$ es\&sour ce $=$ gbs_ge_summary_r\&cad $=0 \# \mathrm{v}=$ onep age\&q\&f=false (03/01/2016, 20:20).

- (2006), «Greek Prepositions: Patterns of Polysemization and Semantic Bleaching», en E. Crespo - J. de la Villa \& A.R. Revuelta (eds.), Word Classes and Related Topics in Ancient Greek. Proceedings of the Conference on Greek Syntax and Word Classes held in Madrid on 18-21, June 2003. Louvain-La-Neuve, Peeters: 487-499.

- $\left(2013^{\mathrm{a}}\right)$, «Adpositional Phrase», en Georgios K. Giannakis [ed.], EAGLL, [en línea] (Koninklijke, Brill NV), en http://referenceworks.brillonline.com/entries/encyclopediaof-ancient-greek-language-and-linguistics/adpositional-phrase-COM_00000006 $(15 / 01 / 2016,19: 04)$.

- $\left(2013^{\mathrm{b}}\right)$, «Agency and Causation», en Georgios K. Giannakis [ed.], EAGLL, [en línea] (Koninklijke, Brill NV), en http://referenceworks.brillonline.com/entries/encyclopediaof-ancient-greek-language-and-linguistics/adpositional-phrase-COM_00000006 (14/03/2016, 11:19). 
Martínez Vázquez, Rafael (1999), \& al., Gramática funcional-cognitiva del griego antiguo I. Sintaxis y semántica de la predicación, Sevilla, Univ. de Sevilla.

- $\left(2000^{\mathrm{a}}\right)$, «Sobre la distinción entre Agente y Causa en griego antiguo», Habis 32: 639-657.

- $\left(2000^{\mathrm{b}}\right)$, «La función semántica "agente” en griego antiguo», Habis 31: 481-502.

Mateos, Juan (1989), Método de análisis semántico aplicado al griego del Nuevo Testamento, Córdoba, El Almendro.

— \& Peláez, Jesús \& Gasco (2000-), DGENT. Fasc. 1-5, Córdoba, El Almendro.

— \& Schökel, Alonso (eds.) (2001 3 ), Nuevo Testamento, Córdoba, El Almendro.

Moulton James H. (ed.), (1963), A Grammar of New Testament Greek. Vol. 3. Syntax, by N. Turner, Edimburgo, T. \& T. Clark.

Nácar, Eloíno \& Colunga, Alberto (1999), Sagrada Biblia, Madrid, B.A.C.

Nelson, Thomas (ed.), (1982), Holy Bible. New King James Version, [en línea] (Biblegateway), en https://www.biblegateway.com/versions/King-James-Version-KJV-Bible/ (15/03/2016, 17:59).

Peláez, Jesús (1996), Metodología del Diccionario Griego-Español del Nuevo Testamento, Córdoba, El Almendro.

Reina, Casiodoro de \& Valera, Cipriano de (eds.) (2010), Santa Biblia [en línea] (Biblegateway), en https://www.biblegateway.com/versions/Reina-Valera-1960-RVR1960-Biblia/ (17/03/2016,17:58).

Robertson, Archibald T. (1914), A Grammar of the Greek New Testament in the Light of Historical Research, Nashville, Broadman.

Rodríguez Adrados, Francisco (2005), «Reflexiones sobre los sistemas de preposiciones del griego antiguo a partir del DGE», en F. Rodríguez Adrados \& J. Rodríguez Somolinos (eds.), La lexicografía griega y el Diccionario griego-español Diccionario griegoespañol. Anejo VI. Madrid, CSIC: 235-244.

— \& al. (2013), DGE [en línea] (Madrid, CSIC), en http://dge.cchs.csic.es/xdge/ả $\pi$ ó $(07 / 03 / 2016,20: 20)$.

Thayer, Joseph H. \& al. (1889²), Greek-English Lexicon of the New Testament, en Bibleworks 7 [CD-ROM], Norfolk, VA 2006, BibleWorks, Software Program.

The Division of Christian Education of the National Council of the Churches of Christ in the United States of America (1989), The Bible. New Revised Standar Version, [en línea] (Biblegateway), en https://www.biblegateway.com/versions/New-Revised-StandardVersion-NRSV-Bible/ (15/03/2016, 17:59).

Wallace, Daniel B. (1996), Greek Grammar Beyond the Basics: An Exegetical Syntax of the New Testament, Grands Rapids, Michigan, Zondervan.

Zerwick, Max (1979), El griego del Nuevo Testamento, Navarra, Verbo Divino.

- (2008), Análisis gramatical del griego del Nuevo Testamento, Navarra, Verbo Divino.

Zorell, Francisco $\left(1990^{4}\right)$, Lexicon Graecum Novi Testamenti, Paris, P. Lethielleux. 